\title{
Control Methods Using Cross-Coupling Effects for Suppression of Rotor/Stator Rubbing System
}

\author{
Zhiyong Shang ${ }^{1, a}$, Haiying Zhang ${ }^{2}$, Talgar shaymurat ${ }^{1}$ \\ ${ }^{1}$ Key Laboratory of New Energy and Materials Research ,Xinjiang Institute of Engineering, China \\ 2 Xinjiang Career Technical College of Sports, China
}

\begin{abstract}
. this paper investigated the influence of cross-coupling effects on the rubbing-related dynamics of rotor/stator systems, The stability analysis on the synchronous full annular rub solution of a rotor/stator system, which includes both the dynamics of the stator and the deformation on the contact surface as well as the cross-coupling terms in velocities and displacements, is carried out. It is found that some cross-coupling effects will benefit the synchronous full annular rubs and some will not. Based on the finding, a control method by generating cross-coupling damping on the stator through the active auxiliary bearing is then proposed in order to suppress the contact severity and avoid the rubbing instability. Numerical simulation shows the validity of the mehtod.
\end{abstract}

\section{Introduction}

In order to improve the efficiency, the gap between the rotor and the stator of rotating machine is setting smaller and smaller, while the rotor/stator rubbing becomes more and more common. So the rotor/stator rubbing is a serious malfunction in the operation of a rotating machine, which can seriously degrade the machine performance, sometime can even lead to disastrous malfunction of the machine.

There are a large amount of works on phenomena of the rotor/stator rubbing system, in order to get the deep insights into the system dynamical behaviors and their relationship with the system parameters. It becomes well known that rotor/stator rubbing can induce different kinds of dynamical behaviours, the periodic synchronous full annular rubs[1-4], sub-and super-harmonic motions [5,6], the quasi-periodic partial rubs[7,8], chaotic responses $[9,10]$, as well as the destructive self-excited dry friction backward whirl $[2,11,12]$.

Since rotor/stator system rubbing is caused by some malfunctions that usually induce the rotor have large deflections, which will cover the clearance between the rotor and the stator, so suppress the vibration amplitude of the rotor is an effective way to prevent from the rotorto-stator rubbing, i.e., through active controlled journal bearings or active magnetic bearing [13-15].

When the rotor and the stator rub is unavoidable, i.e., without the measures for vibration suppression or the failure of the measures in some extreme destructive condition, we still expected that the rotating system can adapt the changing environments and optimize its performance to reduce the rotor and stator rub behaviors

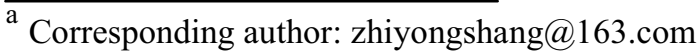

which can induce degradation and, especially, to avoid the occurrence of the destructive instability.

In this paper the interaction effects between the dry friction and the cross-coupling effects during the rotor-tostator rubbing is investigated. By using more realistic effects in the rotor/stator system model, study the influence on the rubbing behaviors of the system; on the other hand, try to use these interaction effects to develope new control methods to reduce the rubbing severity. This paper is arranged as the follows. In Section 2 the model of the rotor/stator system including both the dynamics of the stator (auxiliary bearing) and the deformation on the contact surface as well as the cross-coupling effects is introduced. In Section 3 it demonstrate the influence of the cross-coupling effects on the stability of the synchronous full annular rub solution. Based on the results of the stability analysis of synchronous full annular rub solution, the controllers generating proper cross-coupling effects to reduce the rubbing severity are developed in Section 4. And in Section 5, the conclusions are drawn..

\section{The Rotor/Stator Model with Cross- coupling Effects}

The schematic of the rotor/stator model studied in this paper[12] is shown in Figure 1. A weightless shaft supported by two ideal bearings has effective transverse stiffness $k_{r}$ and rotates at an angular speed $\omega$. A rigid disk of mass $m_{r}$ is mounted at the midpoint of the shaft. Concentric with the disk is an annular stator (an auxiliary bearing) of mass $m_{s}$. The stator is elastically supported by a symmetrical set of springs with isotropic radial stiffness 
$k_{s}$. A clearance $\delta$ between the rotor and the stator. Consider the deformation at the contact surface between the rotor and stator, a symmetrical set of fictive springs with isotropic radial stiffness $k_{c}$ is assumed being laid in the inner ring of the stator to model the contact stiffness. The mass eccentricity of rotor is $e$.

The equations that govern the motion of the system in the complex form can be written as:

$$
\begin{aligned}
& m_{r} \ddot{r}_{r}+\left(c_{r}-j \gamma_{r}\right) \dot{r}_{r}+\left(k_{r}-j Q_{r}\right) r_{r}+\Theta F=m_{r} e \omega^{2} e^{j \omega t} \\
& m_{s} \ddot{r}_{s}+\left(c_{s}-j \gamma_{s}\right) \dot{r}_{s}+\left(k_{s}-j Q_{s}\right) r_{s}-\Theta F=0 \\
& F=k_{c}(1+j \mu)\left(r_{r}-r_{s}-\delta \frac{r_{r}-r_{s}}{\left|r_{r}-r_{s}\right|}\right)
\end{aligned}
$$

In equation (1), $r_{r}=y_{r}+j z_{r}$ and $r_{s}=y_{s}+j z_{s}$ are the complex deflection, $\mu$ is the friction coefficient. $c_{r}$ and $c_{s}$ are the damping of the rotor and the stator respectively. $F$ is the resultant contact force on the contact surface. $\gamma_{r}$ and $\gamma_{s}$ are the cross-coupling damping terms of the rotor and the stator, $Q_{r}$ and $Q_{s}$ the cross-coupling stiffness terms of the rotor and the stator, respectively, to represent the crosscoupling effects. Additionally, we define $\Theta=1$, if $\left|r_{r}-r_{s}\right| \geq \delta$ and $\Theta=0$, if $\left|r_{r}-r_{s}\right|<\delta$.

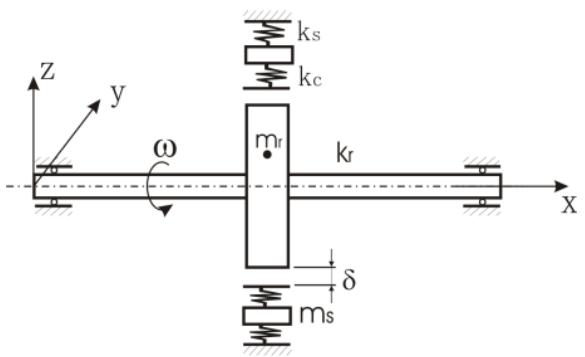

Figure 1(a). The schematic plot of the rotor-to-stator system;

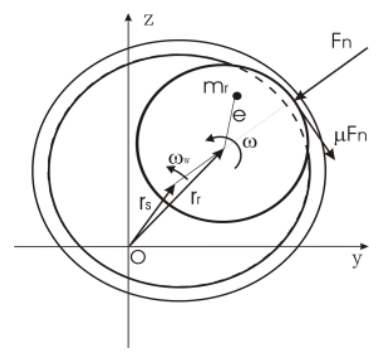

Figure 1(b).the section view on the plane of the rotor/stator ring.

When $\Theta=0$ in Eqn. (1). the rotor/stator system is a linear system without rubbing, this governing equation has steady-state periodic solution, but the cross-coupling stiffness may induce the instability of the system when the cross-coupling stiffness goes above a critical value. From reference[12], it has been shown that for a rotor system with rubbing, the dry friction at the contact surface can induce the instability of the rotor/stator system when the dry friction exceeds a critical value.

When $\Theta=1$ in Eqn. (1), the Eqn. (1) has a steadystate periodic solution-the synchronous full annular rub solution which has constant amplitude and frequency that equals to the rotating speed of the rotor. It is well known that only a stable solution corresponds to a physical observable response, so we interest in the stability of the solution. As known the destructive behaviour-dry friction backward whirl of a rotor/stator system always occurs after the synchronous full annular rub loses stability. From reference[16], The stability of synchronous full annular rub solution has been analysised, this analysis has provided some useful information for the design of the rotor/stator system.

\section{The Cross-coupling Effects on the Stability}

To demonstrate the influence of the cross-coupling effects on the stability of the synchronous full annular rub solution, the stability regions of the solution are drawn on the different parameter planes for different values of cross-coupling coefficients. In the following analysis, some system parameters are fixed as $\zeta_{r}=0.05, \zeta_{s}=0.05$, $\beta_{c r}=20.0, \Delta=2.0$ In the following figures, two solid curves $\mathbf{S N}_{\mathbf{l}}$ and $\mathbf{S N}_{\mathbf{r}}$ define the lower and the upper existence boundaries of the synchronous full annular rub solution. Inside the region enclosed by $\mathbf{S N}_{\mathbf{l}}$ and $\mathbf{S N}_{\mathbf{r}}$, the dotted curves show the stability boundaries of the synchronous full annular rub solution.

\subsection{Stability regions on the plane of $\Omega-\mu$}

In this case the mass ratio and the stiffness ratio are fixed to $M_{s r}=0.2, \beta_{\mathrm{sr}}=8.0$. The cross-coupling stiffness of the rotor, $\kappa r$, and the cross-coupling damping of the stator, $\gamma_{\mathrm{s}}$, on the stability regions of the synchronous full annular rub solution on the parameter plane of $\Omega-\mu$ are studied.

Fig.2 (a) shows that with the increase of the crosscoupling stiffness coefficient, $\kappa_{\mathrm{r}}$, the upper stable boundary raises and the stable region of the solution increases, i.e., for $\kappa_{\mathrm{r}}=0.2$. With the further increase of the cross-coupling stiffness coefficient, i.e., to $\kappa_{\mathrm{r}}=0.4$, the upper stable boundary still raises but a lower stable boundary appears. This indicates that the synchronous full annular rub solution becomes unstable in the region below the boundary. Numerical calculation shows that the rotor response is a large amplitude quasi-period full annular rub response. As pointed out in [3] that the dry friction and the cross-coupling stiffness play contrary roles in the stability of the synchronous full annular rub solution. In the above case, it can be easily explained as the cross-coupling stiffness plays a dominant role in the small dry friction range.

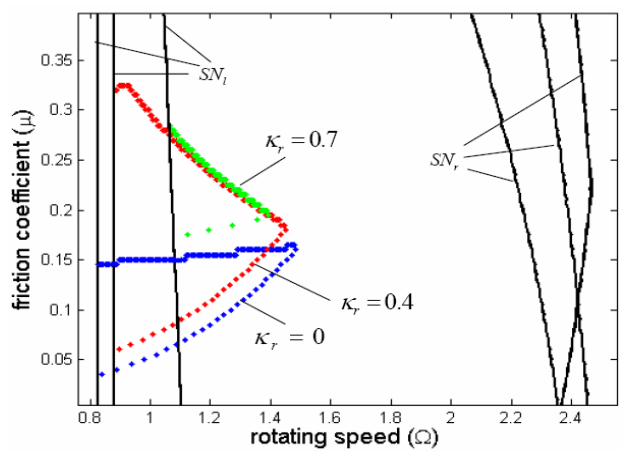

Figure 2(a). Stability chart of synchronous full annular solution in the parameter plane of $\Omega-\mu$. (a): at different cross-coupling stiffness coefficients of the rotor, with $\kappa_{s}=\gamma_{r}=\gamma_{s}=0$. 


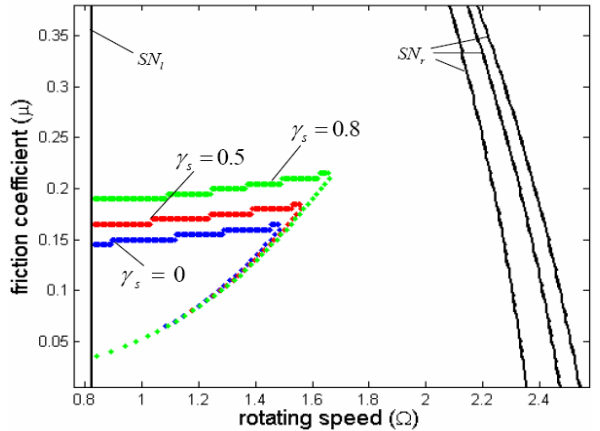

Figure 2(b). at different cross-coupling damping coefficients of the stator, with $\kappa_{r}=\kappa_{s}=\gamma_{r}=0$.

The influence of the cross-coupling damping of the stator on the stability of the synchronous full annular rub solution is demonstrated by Fig.2 (b). It is found that with the increase of the cross-coupling damping coefficient, $\gamma_{\mathrm{s}}$, the stable region of the solution always grows with the upper stable boundary raises. This indicates that the cross-coupling damping on the stator will always benefit the synchronous full annular rub response.

\subsection{Stability regions on the plane of $\Omega-\beta_{s r}$}

In this case the mass ratio and the friction coefficient are fixed to $M_{s r}=0.2, \mu=0.16$. The cross-coupling damping of the rotor, $\gamma_{\mathrm{r}}$, and the cross-coupling stiffness of the stator, $\kappa_{\mathrm{s}}$, on the stability regions of the synchronous full annular rub solution on the parameter plane of $\Omega-\beta_{s r}$ are exploited.

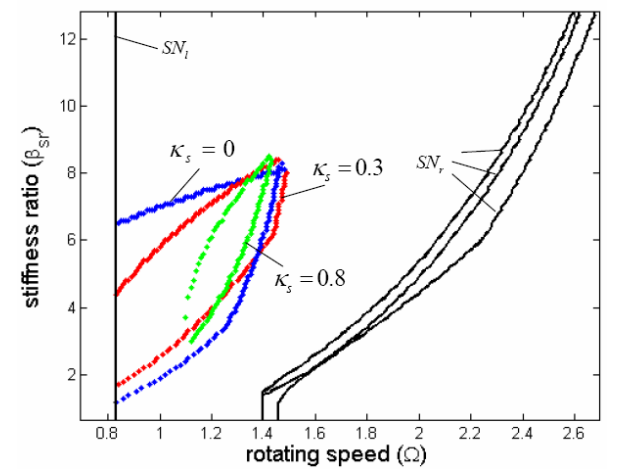

Figure 3(a). Stability chart of synchronous full annular solution in parameter plane of $\Omega-\beta_{s r}$. (a): at different cross-coupling damping coefficients of rotor, with $\gamma_{\mathrm{r}}=\kappa_{s}=\gamma_{s}=0$.

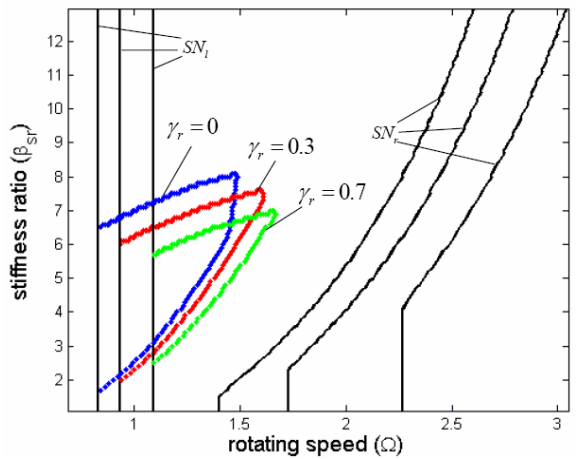

Figure 3(b). at different cross-coupling stiffness coefficients of the stator, with $\kappa_{r}=\gamma_{r}=\kappa_{s}=0$.
It is found from Fig.3 (a) that with the increase of the cross-coupling damping of the rotor, $\kappa_{r}$ the stable boundaries shrink and the stable region of the solution decreases. When $\kappa_{r}=0.8$, the stable region of the solution becomes much smaller than that of $\kappa_{r}=0.3$ and is enclosed by the green curve. This indicates that the cross-coupling damping of the rotor destabilizes the synchronous full annular rub solution.

For the influence of the cross-coupling stiffness of the stator on the stability of the synchronous full annular rub solution, it is easily seen from Fig.3 (b) that with the increase of the cross-coupling stiffness of the stator, $\kappa_{s}$, the boundaries of stable region of the solution always grows, showing the stabilizing effects of the crosscoupling damping on the stator to the synchronous full annular rub response.

\subsection{Results of stability analysis}

It is found through the stability analysis that the crosscoupling damping on the rotor (with $\gamma_{r}>0$ ) and the crosscoupling stiffness on the stator (with $\kappa_{s}>0$ ) in rotor/stator system, if they exist, will not benefit the synchronous full annular rub response of the rotor/stator system because they reduce the stable regions of the synchronous full annular rub solution, or increase the regions for other rubbing behaviors.

However, the cross-coupling stiffness on the rotor (with $\kappa_{r}>0$ ) and the cross-coupling damping on the stator (with $\gamma_{s}>0$ ), if they exist, will generally play a constructive role for the synchronous full annular rub response of the rotor/stator system, as shown by Fig. 4(a) and (b). In the both figures, the stability chart in the system parameter plane of $\Omega-\mu$ is drawn for different value of cross-coupling coefficients. The two solid curves $\mathbf{S N}_{\mathbf{I}}$ and $\mathbf{S N r}$ define the lower and the upper existence boundaries of the synchronous full annular rub solution. In the region left to $\mathbf{S N}_{\mathbf{l}}$ and that right to $\mathbf{S N}_{\mathbf{r}}$, the synchronous no-rub response exists. Inside the region enclosed by $\mathbf{S N}_{\mathbf{l}}$ and $\mathbf{S N}_{\mathbf{r}}$, the dotted curves show the stability boundaries of the synchronous full annular rub solution

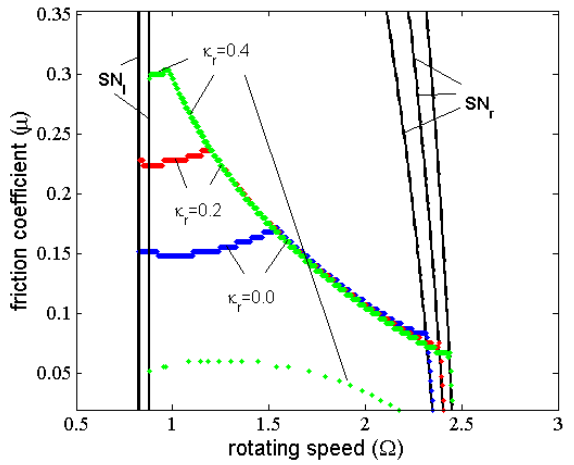

Figure 4(a). Stability chart of synchronous full annular solution in the parameter plane of $\Omega-\mu$. (a): at different cross-coupling stiffness coefficients on the rotor. 


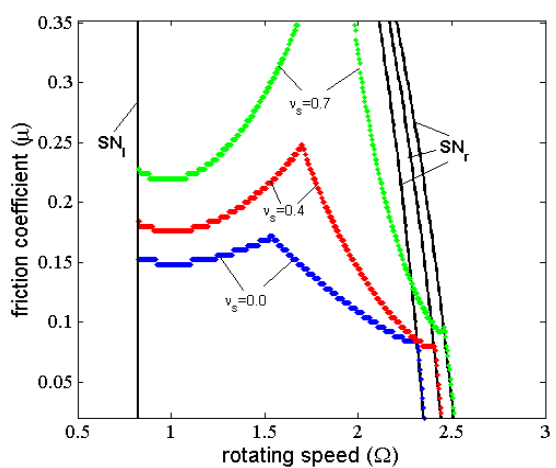

Figure 4(b): at different cross-coupling damping coefficients on the stator.

The influence of the cross-coupling stiffness on the rotor on the stability of the synchronous full annular rub solution is demonstrated by Fig.4 (a). It is found that with the increase of the cross-coupling stiffness coefficient on the rotor, $\kappa_{r}$, the upper stable boundary raises and the stable region of the solution increases, i.e., for $\kappa_{r}=0.2$. With the further increase of the cross-coupling stiffness coefficient, i.e., to $\kappa_{r}=0.4$, the upper stable boundary still raises but a lower stable boundary also appears. This indicateds that the synchronous full annular rub solution becomes also unstable in the region below it. Numerical calculation shows that the rotor response is a large amplitude quasi-period full annular rub response. As known that the dry friction and the cross-coupling stiffness play contrary roles in the stability of the synchronous full annular rub solution. In the above case, it can be easily explained as the cross-coupling stiffness plays a dominant role in the small dry friction range.

The influence of the cross-coupling damping on the stator on the stability of the synchronous full annular rub solution is demonstrated by Fig.4 (b). It is found that with the increase of the cross-coupling damping coefficient on the stator, the stable region of the solution always grows with the upper stable boundary raises. This indicates that the cross-coupling damping on the stator will always benefit the synchronous full annular rub response.

\section{Control Methods for Active Auxiliary Bearings}

Since partial rubs are much more harmful than the synchronous full annular rubs due to their larger contact forces and higher frequencies of vibration. A preliminary goal to reduce the rubbing severity and avoid rubbing instability is to control the rotor/stator system, when rubbing occurs, to the synchronous full annular rub. By using the cross-coupling effects to realize this goal, one could choose to apply the active forces directly on the rotor generating the effect of cross-coupling stiffness, or apply the active forces through stator, i.e., following the idea of active stator control by using active auxiliary bearings. to generate the effect of cross-coupling damping. Based on above stability analysis, it is known that the later method is superior to the former one due to the robust choice of the control parameter and relatively easier way to realize the control forces.
The equations of motion after omitting the cross-coupling terms in the governing equation(1) are written in the matrix form

$$
\mathbf{M} \ddot{\mathbf{q}}+\mathbf{C} \dot{\mathbf{q}}+\mathbf{K q}=\Theta \underline{\mathbf{N}}(\mathbf{q})+\underline{\mathbf{Q}}(\tau)
$$

with $\mathbf{q}=\left(\widehat{y}_{r}, \widehat{z}_{r}, \widehat{y}_{s}, \widehat{z}_{s}\right)^{T}$ and $\mathbf{M}, \mathbf{C}, \mathbf{K}$ being the mass, the damping and the stiffness matrix respectively which can be easily deduced from Eqn.(1). $\mathbf{N}(\mathbf{q})$ is the nonlinear vector containing the components of the resultant contact forces in the corresponding directions. $\mathbf{Q}(\tau)$ is the excitation vector due to unbalance.

For the convenience to control design, Eqn.(2) will be further written in the state space form by introducing state vector $\underline{\mathbf{x}}=\left(\begin{array}{ll}\mathbf{q} & \dot{\mathbf{q}}\end{array}\right)^{T}$ and control vector $\mathbf{u}=\left[\mathrm{u}_{\mathrm{y}}, \mathrm{u}_{\mathrm{z}}\right]^{\mathrm{T}}$ :

$$
\underline{\dot{\mathbf{x}}}=\mathbf{A}_{\mathrm{u}} \underline{\mathbf{x}}+\mathbf{B}_{\mathrm{u}} \underline{\mathbf{u}}+\underline{\mathbf{f}}(\mathbf{t})+\Theta \underline{\mathbf{n}}(\underline{\mathbf{x}})
$$

With:

$$
\begin{aligned}
& \mathbf{A}_{\mathbf{u}}=\left(\begin{array}{cc}
\mathbf{0} & \mathbf{I} \\
-\mathbf{M}^{-1} \mathbf{C} & -\mathbf{M}^{-1} \mathbf{K}
\end{array}\right), \mathbf{B}_{\mathbf{u}}=\left(\begin{array}{c}
\mathbf{0} \\
\mathbf{M}^{-1} \mathbf{H}
\end{array}\right) \\
& \underline{\mathbf{f}}(\tau)=\left(\begin{array}{c}
\mathbf{0} \\
\mathbf{M}^{-1} \underline{\mathbf{Q}}(\tau)
\end{array}\right), \underline{\mathbf{n}(\underline{\mathbf{x}})}=\left(\begin{array}{c}
\mathbf{0} \\
\mathbf{M}^{-1} \underline{\mathbf{N}}(\underline{\mathbf{x}})
\end{array}\right)
\end{aligned}
$$

And $\mathbf{H}$ is a $4 \times 2$ matrix that defines the position of control input.

The cross-coupling damping controller: The development of the controller appears simple because the feedback gain can be directly written in the inertial coordinate system as:

$$
\underline{\mathbf{u}}=-\left[\begin{array}{cc}
0 & -\gamma_{s} \\
\gamma_{s} & 0
\end{array}\right]\left[\begin{array}{l}
\dot{\hat{y}}_{s} \\
\dot{\bar{z}}_{s}
\end{array}\right]=-\mathbf{K C}_{\mathbf{v o l l}} \underline{\mathbf{x}}
$$

Where:

$$
\mathbf{C}_{\text {voll }}=\left[\begin{array}{llllllll}
0 & 0 & 0 & 0 & 0 & 0 & 1 & 0 \\
0 & 0 & 0 & 0 & 0 & 0 & 0 & 1
\end{array}\right], \mathbf{H}^{T}=\left[\begin{array}{llll}
0 & 0 & 1 & 0 \\
0 & 0 & 0 & 1
\end{array}\right]
$$

Below an example is given to show how the control parameters are determined and what results the controller can achieve, with $\mu=0.15$ and $\Omega=1.90$.

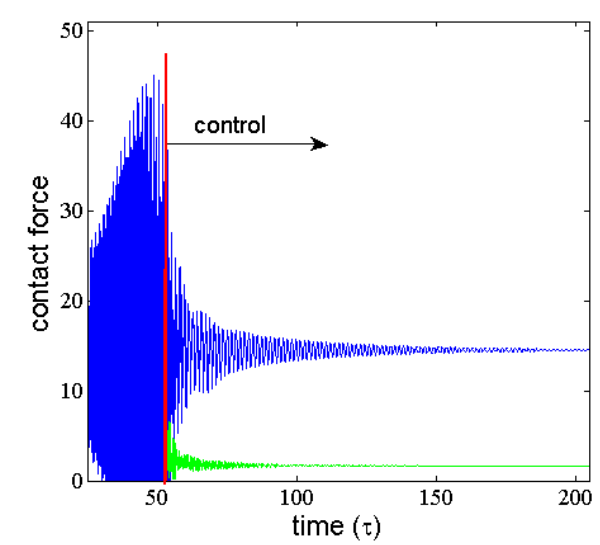

Figure 5(a). The results of controller with $\mu=0.15, \Omega=1.90$. (a): The time history of the resultant contact force (black) and the resultant control force (gray). Right: the time history of the rotor deflection in the y direction. 


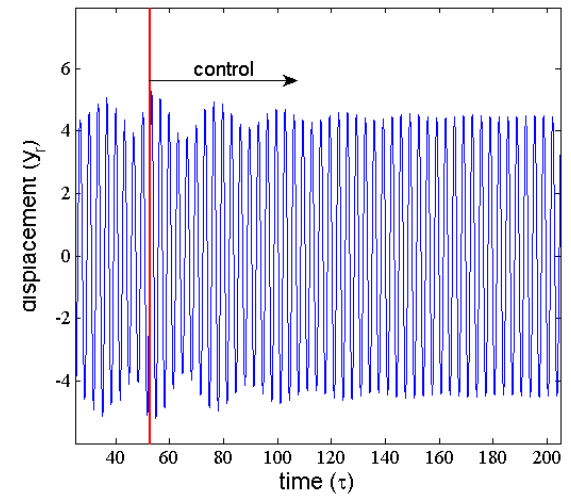

Figure 5(b): time history of rotor deflection in the $\mathbf{y}$ direction.

It is found from Fig.4 that the synchronous full annular rub solution is unstable at the parameters. The partial rub response in this case induces large contact forces to about 40 and a large amplitude vibration of the rotor to about 5 (see Fig.5). From the stability analysis, it is known that when the cross-coupling damping on the stator $\gamma_{s}$ is increased to about 0.4 , the synchronous full annular rub solution will become stable, that means the response of the rotor/stator system will be in the synchronous full annular rub at the given rotating speed.

Based on the above observation, a controller is then designed by choosing $\gamma_{s}=0.4$ to generate the crosscoupling damping on the stator in order to stabilize the system to the synchronous full annular rub. After switching on the controller at 15 th rotation, the rotor responses is stabilized to the synchronous full annular rub quickly and smoothly As seen from the Fig.5(a) the contact force has been significantly reduced. In this case the amplitude of the rotor has an observable increase (see the Fig.5(b)). Again the maximal control forces in each direction are kept under the given limit $(<3)$. In comparison with the optimal controller given in [17] with the same parameters (see Figure 5 in [17]), the present controller achieves a better results with smaller control forces.

\section{Conclusions}

To study the influence of cross-coupling effects of the rotor/stator rubbing system, we use a rotor/stator system which including the dynamics of rotor and stator, and the deformation of contact surface with the cross-coupling damping and stiffness. It is shown that the cross-coupling damping on the rotor and the cross-coupling stiffness on the stator will reduce the regions of the synchronous full annular rubs and can induce system becoming heavy rubbing. The cross-coupling stiffness on the rotor will benefit for synchronous full annular rub in the range of large dry friction and induces the instability in the range of small dry friction. On the other hand, the crosscoupling damping on the stator will always benefit for the synchrounous full annular rubs. Based on the finding, a control method by generating cross-coupling damping on the stator through the active auxiliary bearing is proposed in order to suppress the contact severity and avoid the rubbing instability. Numerical simulation shows the validity of the mehtod and the achievement of better results in comparing with the optimal controller.

Acknowledgments:The authors are grateful for the financial support by the Xinjiang Young scientific and Technological Innovation Talents Project under the grant No. 2014731030 and the National Natural Science Foundation of China, No.51403180

\section{References}

1. Muszynska A, Sound and Vib. Digest, 21, 3-11 (1989).

2. Black HF, International J. of Mech. Eng. Sci., 10, 112(1968).

3. Jiang J and Ulbrich H, Nonli. Dyn., 24,269283(2001).

4. Yu JJ, Goldman P, Bently DE and Muszynska A, ASME J. Gas Turbine \& Power, 124 340-350(2002).

5. Childs DW, J. of Mech. Design, 101, 640-644(1979).

6. Ehrich FF, ASME J. Vib. Acoustics, Stress \& Reliability, 110, 9-16(1988),.

7. Day WB, Quart. Appl. Math., 44, 779-792(1987).

8. Y.B. Kim and S.T. Noah, J. of Sound And Vibration, 190, 239-253 (1996).

9. S.K. Choi and S.T. Noah, ASME J. of Applied Mechanics, 61, 131-138 (1994).

10. M.F. Abdul Azeez and A.F. Vakakis, Int. J. of Nonli. Mechanics, 34, 415-435 (1999).

11. Choi YS, , J.Sound \& Vib., 258, 191-198 (2002).

12. Jiang J and Ulbrich H, ASME J. Acoustics \& Vib., 127, 594-603(2005).

13. Sun L, Krodkiewski JM, J. Sound \&Vib., 213, 114(1998).

14. Knospe C, Hope R, Tamer S and Fedigan S, J.Vib. \& Control, 2, 33-52 (1996)

15. Qiu JH, Tani J and Kwon T, ASME J. Vib. \& Acoustics, 125 328-334(2003).

16. ZhiYong S, Talgar S, Appl.Mech. and Materials, vol. 614, pp48-52 (2014)

17. Jiang J, Ulbrich H, Chavz A, Int. J. Non-Linear Mech., 41/8: 949-957. (2006) 